\title{
A Construction of almost all Brauer Trees
}

\author{
Natalie Naehrig \\ Lehrstuhl D für Mathematik \\ RWTH Aachen \\ Templergraben 64 \\ 52062 Aachen \\ naehrig@math.rwth-aachen.de
}

February 19, 2007

\begin{abstract}
We know that almost all Brauer trees are shaped like a star. Given such a star and an odd prime $l$, we give an explicit method for constructing infinitely many groups with this star as the Brauer tree of some $l$-block. Furthermore we show, that there is an infinite family of Brauer trees which cannot be realized in the principal block of any group.
\end{abstract}

\section{Introduction}

W. Feit showed in his paper [Fei84], that every Brauer tree is similar to an 'unfolded' Brauer tree of a covering group of a finite simple group. Feit calls two Brauer trees similar if both are unfoldings of a third Brauer tree around its exceptional vertex. Moreover he proved using the classification of the finite simple groups, that a Brauer tree of a covering group of a finite simple group either has at most 248 edges or is a straight line. On the one hand, in [FS90],[FS84],[FS82] P. Fong and B. Srinivasan analysed Brauer trees for a given prime $l$ in classical groups and showed that they are straight lines with the exceptional vertex sitting on a position depending on the chosen parameters. On the other hand we know that the alternating groups have straight lines as Brauer trees, too. Hence 'most' Brauer trees are unfolded straight lines, i.e. stars as in Figure 1.

In this paper we investigate the class of star shaped Brauer trees. Before we formulate our main results, we give a precise definition.

Definition 1.1 Let $s$ be a nonnegative integer and $t, f$ be positive integers. A star shaped Brauer tree $S_{s, t, f}$ as in Figure 1 is a tree with $f$ rays with $s$ edges and $f$ rays with $t$ edges, on the understanding that there are only $f$ rays with $t$ edges if $s=0$. If $f>1$, the exceptional character, if it exists, is located in the center of this star. If $f=1$, we assume that an exceptional character exists and that $s$ edges are on one side and $t$ edges are on the other side of the exceptional vertex. We call $S_{s, t, 1}$ a basic tree.

Given any star as in Figure 1 we give methods for constructing groups with this star as a Brauer tree. To be more precise we prove the following.

Theorem 1 Let $l$ be an odd prime. Let $s$ be a nonnegative integer and let $\delta, t, f$ be positve integers, such that $e f \mid l-1$, where $e:=s+t$. Then there are infinitely many groups having a cyclic $l$-block of defect $\delta$ with Brauer tree $S_{s, t, f}$.

More specifically, we have the following. 


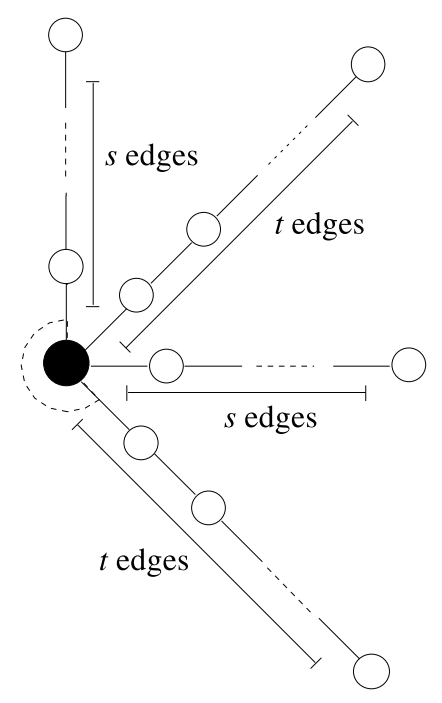

Figure 1: $S_{s, t, f}$

(a) If $s=0$, hence $e=t$, there are infinitely many primes $p$ such that the principal l-block of $G L_{e}\left(p^{f}\right) \rtimes\langle\sigma\rangle$ has a cyclic block of defect $\delta$ and has Brauer tree $S_{0, e, f}$. Here, $\sigma$ denotes the field automorphism of order $f$ acting on $G L_{e}\left(p^{f}\right)$.

(b) If $s, t$ are positive and if $e:=s+t$ is odd, there are infinitely many primes $p$ and a positive integer $n$ such that $G U_{n}\left(p^{f^{\prime}}\right) \rtimes\langle\sigma\rangle$ has a cyclic $l$-block of defect $\delta$ with Brauer tree $S_{s, t, f}$. Here we let

$$
f^{\prime}:=\left\{\begin{aligned}
f, & \text { if } f \text { is odd, } \\
f / 2, & \text { if } f \text { is even, }
\end{aligned}\right.
$$

and $\sigma$ be the field automorphism of order $f$ acting on $G U_{n}\left(p^{f^{\prime}}\right)$.

(c) If $s, t$ are positive and if $e:=s+t$ is even, then there are infinitely many primes $p$ and a positive integer $m$ such that $G_{2 m}\left(p^{f}\right) \rtimes\langle\sigma\rangle$ has a cyclic $l$-block of defect $\delta$ with Brauer tree $S_{s, t, f}$. Here $G_{2 m}\left(p^{f}\right)$ denotes one of the groups $S p_{2 m}\left(p^{f}\right), S O_{2 m}^{+}\left(p^{f}\right)$ or $S O_{2 m}^{-}\left(p^{f}\right)$ and $\sigma$ the field autmorphism of order $f$ acting on $G_{2 m}\left(p^{f}\right)$.

In each case the star $S_{s, t, f}$ is the planar embedded Brauer tree of the block.

After this constructive part we turn to the question whether every Brauer tree is realizable in the principal block of some group. We show that it suffices to answer this question for simple groups. Theorem 2 gives an infinite family of Brauer trees which do not occur in any principal $l$-block.

Theorem 2 With the notation of Theorem 1, let $s, t$ be positive integers with $s \neq t$ and $s+t>248$. Then $S_{s, t, f}$ is not the Brauer tree of any principal $l$-block.

By reducing the assertion of Theorem 2 to finite simple groups, we need the classification for its proof. Since we do not know the shape of the Brauer trees of the principal blocks of $E_{7}(q)$ and $E_{8}(q)$, we can only use the fact, that the number of edges is less than 249 in these cases. This is the reason for restricting the class of Brauer trees in Theorem 2. In fact, as we will see in Section 6, there are only a few exceptions, where a principal $l$-block is an irregularly shaped star with less than 249 edges.

In Section 2 we ensure the existence of suitable primes needed as characteristics of fields in subsequent chapters. In Sections 3, 4 and 5 we construct groups having an 
l-block with the desired Brauer tree. The question whether there are possible Brauer trees, which cannot be realized in the principal block of some group is analysed in Section 6. First we prove that it suffices to consider Brauer trees of simple groups and then examine the Brauer trees of the principal blocks of simple groups.

\section{Primes and More}

In Sections 3, 4 and 5 we will examine classical groups over finite fields whose characteristic must satisfy several conditions. Here we show that such primes exist and that there are infinitely many of them. Throughout this paper we fix an odd prime $l$ and let $\bar{x}$ denote the residue class of $x$ in $\mathbb{Z} / l \mathbb{Z}$ for $x \in \mathbb{Z}$ and $|\bar{x}|_{l}$ the order of $\bar{x}$ in $\mathbb{Z} / l \mathbb{Z}$, if $l \nmid x$. We further denote the $l$-adic valuation by $\nu_{l}$.

Proposition 2.1 Let $l$ be an odd prime, and let $\delta, k$ be positive integers such that $k \mid l-1$. Then there are infinitely many primes $p$ satisfying

$$
\begin{aligned}
& l^{\delta} \mid p^{k}-1, \\
& l^{\delta+1} \nmid p^{k}-1, \\
& l \nmid p^{i}-1 \text { for } 1 \leq i \leq k-1 .
\end{aligned}
$$

Proof: Let $a^{\prime} \in \mathbb{Z}$ be such that $\left|a^{\prime}\right|_{l}=k$. If $\nu_{l}\left(\left(a^{\prime}\right)^{k}-1\right)=1$, we set $a^{\prime \prime}:=a^{\prime}$. Else we put $a^{\prime \prime}:=a^{\prime}+l$ and observe that $\left|\overline{a^{\prime \prime}}\right|_{l}=k$ and $l^{2} \nmid\left(a^{\prime \prime}\right)^{k}-1$. By [HupBla82, La 8.1], $\nu_{l}\left(a^{k}-1\right)=\delta$ with $a:=\left(a^{\prime \prime}\right)^{l^{\delta-1}}$. We apply Dirichlet's Theorem ([Has64, p.176]) to $l^{\delta+1}$ and $a$, and find infinitely many $k_{0}$ in $\mathbb{N}$ such that $a+k_{0} l^{\delta+1}$ are primes which satisfy (1).

\section{The Regularly Shaped Star}

\subsection{The Basic Tree}

With the notation of the Main Theorem we let $s=0$, i.e., $e=t$, and $f$ be a positive integer such that ef $\mid l-1$. We apply Proposition 2.1 to $k=e f$, fix one of the primes and denote it by $p$. Hence,

$$
l \mid p^{e f}-1 \text { and } l \nmid p^{i}-1 \text { for all } 1 \leq i \leq e f-1 .
$$

Put $q:=p^{f}$ and denote the general linear group $G L_{e}(q)$ by $G$. Further let $T_{0} \leq G$ be a Coxeter torus (see [Hup67, p.187]) and $D_{0} \leq T_{0}$ be the Sylow $l$-subgroup of $T_{0}$. Then $D_{0}$ is also a Sylow $l$-subgroup of $G$.

Lemma 3.1 We have $N_{G}\left(D_{0}\right)=N_{G}\left(T_{0}\right)$ and $C_{G}\left(T_{0}\right)=T_{0}=C_{G}\left(D_{0}\right)$.

Proof: For the first two equalities see [Hup67, pp. 187, Satz 7.3]. Let $t_{0} \in T_{0}$ with $\left\langle t_{0}\right\rangle=T_{0}$. Then the eigenvalues of $t_{0}$ (in an algebraic closure $\overline{\mathbb{F}}_{q}$ of $\mathbb{F}_{q}$ ) are $a, a^{q}, \ldots$, $a^{q^{e-1}}$, where $a$ is a generator of $\mathbb{F}_{q^{e}}^{*}$. For a suitable $r \in \mathbb{N}$ let $z_{0}:=t_{0}^{r} \in D_{0}$ be an element of order $l$. Then the eigenvalues of $z_{0}$ are $a^{r}, a^{r q}, \ldots, a^{r q^{e-1}}$. Because of assumption (2) these eigenvalues are pairwise distinct. It follows that any element in $G L_{e}\left(\overline{\mathbb{F}}_{q}\right)$ which commutes with $z_{0}$ also commutes with $t_{0}$. Since $C_{G}\left(t_{0}\right)=T_{0}$ the claim follows.

By the Schur-Zassenhaus Theorem we have $C_{G}\left(D_{0}\right)=T_{0}=D_{0} \times L \unlhd N_{G}\left(D_{0}\right)$. Fix the principal block of $C_{G}\left(D_{0}\right)$ which has $D_{0}$ as a defect group and consider its canonical character $1_{D_{0}} \otimes 1_{L}$. This character corresponds uniquely to the principal block $b_{0}$ of $N_{G}\left(D_{0}\right)$ by Theorem [Alp93, Thm. 15.1(5)]. By Brauer's First Main Theorem $b_{0}$ corresponds to the principal block of $G$ (with defect group $D_{0}$ ). From [FS84] we know that the Brauer tree of the principal block of $G$ is of the form 


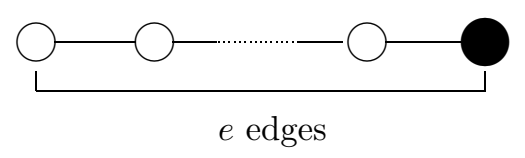

with the exceptional vertex at one end. To obtain the star $S_{0, e, f}$, it remains to unfold the basic tree by extending $G$.

\subsection{Unfolding the Basic Tree}

Let $\sigma: G \longrightarrow G,\left[g_{i j}\right] \mapsto\left[g_{i j}^{p}\right]$ be the restricted Frobenius automorphism of $G$ and let $\hat{G}:=G \rtimes\langle\sigma\rangle$. Note that $|\hat{G}|=|G| \cdot f$, hence $D_{0}$ is a Sylow $l$-subgroup of $\hat{G}$. We examine what happens to the centralizer and normalizer of $D_{0}$ when extending $G$.

Lemma 3.2 Let $D_{0}, T_{0}$ be as above.

(a) We have $C_{\hat{G}}\left(D_{0}\right)=T_{0}$.

(b) We have $\left|N_{\hat{G}}\left(D_{0}\right): N_{G}\left(D_{0}\right)\right|=f$.

Proof:

(a) Let $t_{0} \in T_{0}$ be a generator of $D_{0}, z_{0}:=t_{0}^{r} \in D_{0}$ be an element of order $l$ with eigenvalues $a^{r}, a^{r q}, \ldots, a^{r q^{e-1}}$ for a generator $a$ of $F_{q^{e}}^{*}$. Let $g \in G$ and $0 \leq i \leq f-1$. Then $\left(g, \sigma^{i}\right) \in \hat{G}$ centralizes $\left(z_{0}, 1\right)$ if and only if $\sigma^{i}\left(z_{0}\right)=g^{-1} z_{0} g$.

Assume $\left(g, \sigma^{i}\right)$ with $i>0$ centralizes $z_{0}$. On the one hand $g^{-1} z_{0} g$ has the same eigenvalues as $z_{0}$. On the other hand, $\sigma^{i}\left(z_{0}\right)$ has eigenvalues $a^{r p^{i}}$, $\left(a^{r p^{i}}\right)^{q}, \ldots,\left(a^{r p^{i}}\right)^{q^{e-1}}$. Hence $a^{r}=\left(a^{r p^{i}}\right)^{q^{k_{0}}}$ and $l \mid p^{i+f k_{0}}-1$ for some $0 \leq k_{0} \leq e-1$, which contradicts (2). Thus the assumption is wrong.

(b) Let $N:=N_{G}\left(D_{0}\right)$ and $\hat{N}:=N_{\hat{G}}\left(D_{0}\right)$. By the Frattini argument we have $\hat{G}=G \hat{N}$, hence $\hat{G} / G=G \hat{N} / G \cong \hat{N} /(G \cap \hat{N})=\hat{N} / N$. As $\hat{G} / G$ is cyclic of order $f$ we get the assertion.

As $1_{D_{0}} \otimes 1_{L}$ is invariant in $N_{\hat{G}}\left(D_{0}\right)$ the inertia index of the principal block of $\hat{G}$ is

$$
e_{\hat{G}}=\left|T_{N_{\hat{G}}\left(D_{0}\right)}\left(1_{D_{0}} \otimes 1_{L}\right): C_{\hat{G}}\left(D_{0}\right)\right|=\left|N_{\hat{G}}\left(D_{0}\right): C_{\hat{G}}\left(D_{0}\right)\right|=f e .
$$

Hence the Brauer tree of the principal block of $\hat{G}$ has the desired number of edges. From [Fei84, La 3.2] we know that the basic tree opens at the exceptional vertex while expanding $G$ to $\hat{G}$.

\section{The Irregularly Shaped Star I}

Throughout this section we let $l$ be an odd prime and $s, t, f$ be positive integers such that $s+t=: e$ is odd and ef $\mid l-1$. Put

$$
f^{\prime}:= \begin{cases}f, & \text { if } f \text { is odd, } \\ f / 2, & \text { if } f \text { is even. }\end{cases}
$$

Note that $2 e f^{\prime} \mid l-1$. We then apply Proposition 2.1 to $k=2 e f^{\prime}$, fix one of the primes and denote it by $p$. Hence,

$$
l \mid p^{2 e f^{\prime}}-1 \text { and } l \nmid p^{i}-1 \text { for all } 1 \leq i \leq 2 e f^{\prime}-1 .
$$

We put $q:=p^{f^{\prime}}$ and denote by $G_{n}:=G U_{n}(q)$ the general unitary group over $\mathbb{F}_{q^{2}}$, i.e., the matrix group $G_{n}:=\left\{x \in G L_{n}\left(q^{2}\right) \mid x \bar{x}^{t r}=E_{n}\right\}$, where $\bar{x}$ is the matrix obtained by raising each entry to its $q$ th power. By condition (3), we have $l \mid q^{e}+1$ and $l \nmid q^{j}+1$ for $1 \leq j \leq e-1$. Hence, $|-q|_{l}=e$ and $|q|_{l}=2 e$ and by [FS90, Sec.2], $l$ is unitary for $G_{n}$. 


\subsection{Theoretical Background}

Let us first recall some background information which will be of great use later.

Definition 4.1 Let $m$ be a positive integer.

(a) A partition of $m$ is a finite sequence $\lambda:=\left(a_{1}, \ldots, a_{k}\right) \in \mathbb{N}_{0}^{k}$ such that $a_{i} \leq a_{i+1}$ and $\sum_{i=1}^{k} a_{i}=m$. In this case we write $\lambda \vdash m$. Two partitions are equivalent if their nonzero entries are equal.

(b) Let $X=\left\{x_{1}, \ldots, x_{l}\right\} \subset \mathbb{N}_{0}$ with $x_{1}<\ldots<x_{l}$ and let $\lambda \vdash m$ be a partition. Then $X$ is a $\beta$-set of $\lambda$, if there is an equivalent partition $\lambda^{\prime}=\left(a_{1}^{\prime}, \ldots, a_{l}^{\prime}\right)$ such that $x_{i}:=a_{i}^{\prime}+(i-1)$ for all $1 \leq i \leq l$.

(c) Let $X \subset \mathbb{N}_{0}$ be finite and let $d$ be a nonnegtaive integer. For $d \neq 0$, the $d$-shift of $X$ is the set $\{0,1, \ldots, d-1\} \cup\{x+d \mid x \in X\}$ and for $d=0$ it is $X$ itself. Two $\beta$-sets are equivalent if one set is a $d$-shift of the other for some $d \in \mathbb{N}_{0}$.

(d) A hook $\nu$ of a $\beta$-set $X$ is a pair $(y, x) \in \mathbb{N}_{0}^{2}$ such that $0 \leq y<x$ and $y \notin$ $X, x \in X$. The length of such a hook is $r:=x-y$ and $\nu$ is then an $r$-hook.

(e) Let $\lambda \vdash m$ be a partition, $X$ a $\beta$-set for $\lambda$ and $\nu=(y, x)$ an $r$-hook. We obtain a set $X_{1}:=\{y\} \cup X \backslash\{x\}$ by removing $\nu$. Analogously $X$ is obtained from $X_{1}$ by adding $\nu$. Note that $X_{1}$ is a $\beta$-set for a partition $\lambda_{1} \vdash m-r$. Removing $r$-hooks as often as possible from $X$ will result in a unique $\beta$-set after a finite number of steps, whose corresponding partition is called the $r$-core of $\lambda$.

(f) For $r \in \mathbb{N}$ consider an $r$-abacus consisting of $r$ strings, numbered from left to right by $0,1, \ldots, r-1$ and from top to bottom by $0,1, \ldots$ A position on string $i$ and row $w$ is numbered by $i+w r$. For a partition $\lambda$ and a $\beta$-set $X$ for $\lambda$ we obtain the corresponding abacus by putting a bead on string $i$ and row $t$ $\left(0 \leq i \leq r-1, w \in \mathbb{N}_{0}\right)$ if and only if $i+w r$ is an element of $X$. Note that removing or adding the $r$-hook $(y, x)$ from $X$ means to shuffle up or down the bead belonging to $x$ to the (free) position above or below, respectively. The lowest bead on a string is called index bead.

In our situation we need to consider unipotent blocks and their unipotent characters, which are analysed in [Lu77] or [Lu84].

Theorem 4.2 Let $l$ be unitary for $G_{m}$ and let $e:=|-q|_{l}$.

(a) The unipotent characters of $G_{n}$ are parametrized by the partitions of $n$. We write $\chi_{\lambda}$ for the unipotent character which is parametrized by $\lambda \vdash n$.

(b) Let $\chi_{\lambda}, \chi_{\mu}$ be unipotent characters. Then $\chi_{\lambda}$ and $\chi_{\mu}$ lie in the same block if and only if $\lambda$ and $\chi$ have the same e-core.

Proof: See [FS82].

Lemma 4.3 Let $e$ be odd and $1 \leq s \leq(e-1) / 2$. Then there is a positive integer $n$ and an e-core $\kappa \vdash n-e$ such that the corresponding e-abacus has a bead on each position of the first row, $s$ even and $t:=e-s$ odd index beads. Moreover $\kappa$ is a 2-core.

Proof: Note that two positions, one below the other, are of different parity. First put a bead on each position of the first row. Then put beads on strings 0 to $2 s+1$ on the second row. Further put beads only on odd positions of the remaining strings of the second row. Hence we get the desired number of odd and even index beads. Let $x_{1}<\ldots<x_{k}$ denote the beads in the above theorem. Then $\kappa \vdash \sum_{i=0}^{r} i=: n-e$, with $r=(e-(2 s+1)) / 2$, hence $n-e$ is of the form $r(r+1) / 2$. 
Theorem 4.4 Let $B_{\kappa}$ be a unipotent block of $G_{n}$ with cyclic defect group and corresponding e-core $\kappa \vdash n-e$. Let $X=\left\{x_{1}, \ldots, x_{k}\right\}$ be a $\beta$-set for $\kappa$ and consider the resulting e-abacus of $X$. We assume that there is at least one bead on each string, otherwise we consider an equivalent $\beta$-set. The index beads $\rho_{1}, \ldots \rho_{e}$ will be ordered such that $\rho_{1}>\ldots>\rho_{e}$. The partitions $\lambda_{\rho_{i}}$ obtained by adding the hook $\left(\rho_{i}, \rho_{i}+e\right)$ to $\kappa$ for $1 \leq i \leq$ e label the non-exceptional characters of $B_{\kappa}$.

Proof: [FS90, p.10].

Theorem 4.5 Let $G_{n}, B_{\kappa}, \rho_{1}, \ldots, \rho_{e}$ and $\lambda_{\rho_{i}}, 1 \leq i \leq e$ be as in Theorem 4.4. Denote the subsequence of the even index beads in $\left(\rho_{1}, \ldots, \rho_{e}\right)$ by $\left(\sigma_{1}, \ldots, \sigma_{s}\right)$ and the subsequence of the odd (remaining) beads by $\left(\tau_{1}, \ldots, \tau_{t}\right)$. We obtain two types of partitions in Theorem 4.4:

- $\lambda_{\sigma_{1}}, \ldots, \lambda_{\sigma_{s}}$ (bead $\sigma_{i}$ was shuffled down for $\left.1 \leq i \leq s\right)$,

- $\lambda_{\tau_{1}}, \ldots, \lambda_{\tau_{t}}$ (bead $\tau_{j}$ was shuffled down for $1 \leq j \leq t$ ).

Then the Brauer tree of $B_{\kappa}$ is a basic tree of the following shape with the exceptional character between $\chi_{\sigma_{s}}$ and $\chi_{\tau_{t}}$ :

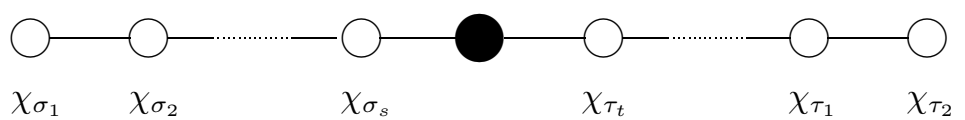

Proof: [FS90, p.21]

The preceeding theorem helps us finding a group with a block having $S_{s, t, f}$ as Brauer tree.

\subsection{The Basic Tree}

Let $n, \kappa$ be as in Lemma 4.3. We proceed to show that $B_{\kappa}$ has a cyclic defect group.

Lemma 4.6 Let $m$ be odd. Then there is a cyclic irreducible subgroup in $G_{m}$ of order $q^{m}+1$, a Coxeter torus of $G_{m}$. Further, each cyclic irreducible subgroup of $G_{m}$ has order dividing $q^{m}+1$.

Proof: [Hup70, p.149].

If $m$ is even, then there is no cyclic irreducible subgroup in $G_{m}$, and we see the reason for distinguishing between $e$ odd and even.

By (3) a Sylow $l$-subgroup of a maximal irreducible subgroup $T \leq G_{e}$ is also a Sylow $l$-subgroup of $G_{e}$. In particular the block $B_{\kappa}$ has a cyclic defect group by [CaEn94].

For the remainder of this section let us fix a Coxeter torus $T_{0}$ of $G_{e}$ and the Sylow l-subgroup $D_{0} \leq T_{0}$. By [Hup67, p.165] we see that there is a Coxeter torus $T_{1}$ of $G L_{e}\left(q^{2}\right)$ with $T_{0} \leq T_{1}$.

Lemma 4.7 Let $G_{e}$ and $T_{0}$ be as above.

(a) We have $C_{G_{e}}\left(T_{0}\right)=T_{0}$ and $C_{G_{n}}\left(T_{0}\right)=T_{0} \times G_{n-e}$.

(b) We have $N_{G_{n}}\left(T_{0}\right)=N_{G_{e}}\left(T_{0}\right) \times G_{n-e}$, where $N_{G_{e}}\left(T_{0}\right)$ is a cyclic extension of $T_{0}$ of order $e$.

(c) We have $C_{G_{n}}\left(D_{0}\right)=T_{0} \times G_{n-e}=C_{G_{n}}\left(T_{0}\right)$.

(d) We have $N_{G_{n}}\left(D_{0}\right)=N_{G_{n}}\left(T_{0}\right)$. 


\section{Proof:}

(a) We have $T_{0}=T_{1} \cap G_{e}$, hence with the assumptions (3) the first assertion follows analogously to Lemma 3.1. As all eigenvalues of a generator $t$ of $T_{0}$ differ from 1 , the second assertion follows by direct calculation of $x t$ and $t x$ for $x \in C_{G L_{n}\left(q^{2}\right)}\left(T_{0}\right)$.

(b) Let $x \in N_{G L_{n}\left(q^{2}\right)}\left(T_{0}\right)$ and $1 \leq r \leq q^{e}+1$ with $\operatorname{gcd}\left(q^{e}+1, r\right)=1$ and $t x=x t^{r}$. Analogously to (a) we get the first equation. For the second see [Ca85, Prop. 3.3.6, La. 3.6.5].

(c) Analogous to (a) and Lemma 3.1.

(d) With relations (3) we follow the proof of Theorem 7.3(1) in [Hup67].

By [Ca85, Sec. 13.7], $G_{n-e}$ has a unique cuspidal character $\chi_{\kappa}$ since $n-e=$ $r(r+1) / 2$. Similar to the end of subsection 3.1 we find the canonical character $1_{T_{0}} \otimes \chi_{\kappa}$ of $B_{\kappa}$, whose Brauer tree is a basic tree.

\subsection{Unfolding the Basic Tree}

We now consider an extension of $G_{n}$ of suitable degree and analyse the corresponding extensions of the blocks and their defect groups under consideration. Obviously the restricted Frobenius automorphism $\alpha:=\bar{\alpha}_{\mid \mathbb{F}_{q^{2}}}$ acts on $G_{n}$. Let

$$
\alpha^{\prime}:= \begin{cases}\alpha^{2}, & \text { if } f=f^{\prime} \text { is odd } \\ \alpha, & \text { if } f=2 f^{\prime} \text { is even. }\end{cases}
$$

Then $\left|\hat{G}_{n}\right|=\left|G_{n}\right| \cdot f$ and $l \nmid f$. As $G_{e}$ and $G_{n-e}$ are invariant under $\alpha^{\prime}$, there exists $g_{0} \in G_{e}$ such that $g_{0}\left(\alpha^{\prime}\left(T_{0}\right)\right) g_{0}^{-1}=T_{0}$. Put $\sigma:=\left(g_{0}, \alpha^{\prime}\right)$. Then $\hat{G}_{n}=G_{n}\langle\sigma\rangle$. Moreover $T_{0}, D_{0}, G_{e}, G_{n-e}, C_{G_{e}}\left(D_{0}\right)$ and $N_{G_{e}}\left(D_{0}\right)$ are invariant under $\sigma$.

Lemma 4.8 We have $C_{\hat{G}_{n}}\left(D_{0}\right)=C_{G_{n}}\left(D_{0}\right)=C_{G_{e}}\left(D_{0}\right) \times G_{n-e}$.

Proof: Analogous to Lemma 3.1 with (3).

By [Alp93, Thm. 1(5)] there is a unique block $\hat{B}$ of $\hat{G}_{n}$ covering $B_{\kappa}$ with $\hat{B}=$ $\left(B_{\kappa}\right)^{\hat{G}_{n}}$.

Lemma 4.9 The cuspidal character $\chi_{\kappa}$ is invariant under $\sigma_{\mid G_{n-e}}$.

Proof: We only need to show the assertion for $\alpha^{\prime \prime}:=\alpha_{\mid G_{n-e}}^{\prime}$. Since the DeligneLusztig varieties (see [Ca85, Sec. 7.7]) are permuted by $\alpha^{\prime \prime}$, we have by [Ca85, Prop. 7.1.5, Thm. 7.7.11]

$$
R_{T_{w, 1}}^{G_{n-e}}\left(\left(\alpha^{\prime \prime}\right)^{-1} g\right)=R_{T_{w, 1}}^{G_{n-e}}(g) \text { for all } g \in G_{n-e},
$$

hence $\alpha^{\prime \prime}$ permutes unipotent characters of $G_{n-e}$ (see [Ca85, Sec.12]). Furthermore, there is an $\alpha^{\prime \prime}$-stable $(B, N)$-pair such that for all Levi subgroups $L$ of $G_{n-e}$

$$
\alpha^{\prime \prime}\left(R_{L}^{G_{n-e}}\left(\chi_{\kappa}\right)\right)=R_{L}^{G_{n-e}}\left(\alpha^{\prime \prime}\left(\chi_{\kappa}\right)\right)
$$

which is 0 by the cuspidality of $\chi_{\kappa}$. By the uniqueness of $\chi_{\kappa}$, the claim follows. We analyse the inertia index of $1_{T_{0}} \otimes \chi_{\kappa}$ :

Lemma 4.10 We have $T_{N_{\hat{G}_{n}}\left(D_{0}\right)}\left(1_{T_{0}} \otimes \chi_{\kappa}\right)=N_{\hat{G}_{n}}\left(D_{0}\right)$. 
Proof: Since $D_{0}$ is invariant under $\sigma, N_{\hat{G}_{n}}\left(D_{0}\right)=\left\langle N_{G_{n}}\left(D_{0}\right), \sigma\right\rangle$. Moreover $1_{T_{0}} \otimes \chi_{\kappa}$ is invariant in $N_{G_{n}}\left(D_{0}\right)$ and $\chi_{\kappa}$ under $\sigma$ by Lemma 4.9 .

Hence the number of edges of the Brauer tree of $\hat{G}_{n}$ is

$$
\left|T_{N_{\hat{G}_{n}}\left(D_{0}\right)}\left(1_{T_{0}} \otimes \chi_{\kappa}\right): C_{\hat{G}_{n}}\left(D_{0}\right)\right|=f \cdot e .
$$

By [Fei84, La. 3.2, La. 4.3], this Brauer tree is an $f$-fold unfolding of the basic tree around its exceptional vertex.

Remark 4.11 The tree in Figure 1 is the planar embedded Brauer tree of the unipotent block $\hat{B}$ : Assume the planar embedding is not as in Figure 1. Then there are two rays of length $s$ sitting next to each other. Let the labelling of the edges of the Brauer trees be as in Figure 2 and Figure 3.

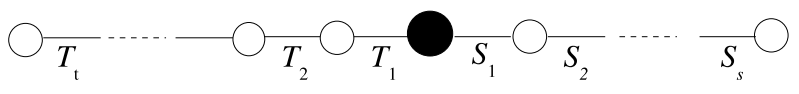

Figure 2: Brauer tree of $B_{\kappa}$

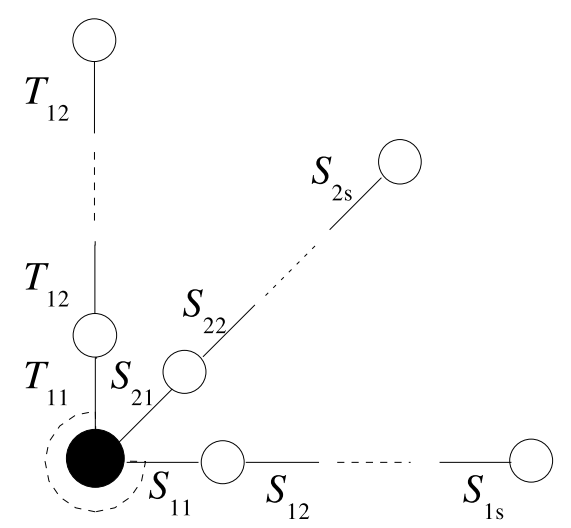

Figure 3: Brauer tree of $\hat{B}$

By [Alp93, Sec. 17] we can deduce the exact structure of the projective indecomposable modules corresponding to the simple modules from the Brauer tree. In particular we know that under the above assumption

$$
\begin{aligned}
& S_{11} \\
& S_{21}
\end{aligned}
$$

is indecomposable as an $\hat{B}$-module. However, the Brauer tree of $B_{\kappa}$ indicates that the restriction of this module to $G_{n}$ decomposes as a direct sum $S_{1} \oplus S_{1}$. This is a contradiction by [HupBla82, Thm. 7.20], as the degree $f$ of the extension does not divide $l$.

\section{$5 \quad$ The Irregularly Shaped Star II}

Throughout this section let $s \neq t, f$ be positive integers such that $s+t=: 2 e^{\prime}=: e$ is even and ef $\mid l-1$. We apply Proposition 2.1 to $k=e f$, fix one of the (odd) primes and denote it by $p$. Hence,

$$
l \mid p^{e f}-1 \quad \text { and } l \nmid p^{i}-1 \text { for all } 1 \leq i \leq e f-1 .
$$


We put $q:=p^{f}$ and denote the $m \times m$ matrix which has 1 on the anti-diagonal and 0 everywhere else by $\mathbf{J}_{m}$. Fix some $a \in \mathbb{F}_{q}$ such that $X^{2}+X+a$ is irreducible in $\mathbb{F}_{q}[X]$ and put

$$
\tilde{\mathbf{J}}_{2 m}=\left[\begin{array}{cc}
0 & \mathbf{J}_{m} \\
-\mathbf{J}_{m} & 0
\end{array}\right] \quad \tilde{\mathbf{J}}_{2 m}^{*}=\left[\begin{array}{llll} 
& & \mathbf{J}_{m-1} \\
& 2 & 1 & \\
& 1 & 2 a &
\end{array}\right] .
$$

By $G_{2 m}$ we then mean one of the following matrix groups:

- The symplectic group $S p_{2 m}(q)=\left\{g \in G L_{2 m}(q) \mid g^{t} \tilde{\mathbf{J}}_{2 m} g=\tilde{\mathbf{J}}_{2 m}\right\}$.

- The special orthogonal group $S O_{2 m}^{+}(q)=\left\{g \in G L_{2 m}(q) \mid g^{t} \mathbf{J}_{2 m} g=\mathbf{J}_{2 m}\right\}$.

- The special orthogonal group $S O_{2 m}^{-}(q)=\left\{g \in G L_{2 m}(q) \mid g^{t} \tilde{\mathbf{J}}_{2 m}^{*} g=\tilde{\mathbf{J}}_{2 m}^{*}\right\}$.

\subsection{Notation and Theoretical Background}

Definition 5.1 (Compare [FS90]) (a) A symbol $\Lambda=\{X, Y\}$ consists of an unordered pair of sets $X$ and $Y$ of nonnegative integers. If $X=Y$, then $\Lambda$ is degenerate. Two symbols $\Lambda=\left\{X_{1}, Y_{1}\right\}$ and $\Lambda_{2}=\left\{X_{2}, Y_{2}\right\}$ are equivalent if there is $d \in \mathbb{N}$ such that

$$
\begin{aligned}
X_{2} & =[0, d-1] \cup\left(X_{1}+d\right) \text { and } Y_{2}=[0, d-1] \cup\left(Y_{1}+d\right) \\
\text { or } X_{1} & =[0, d-1] \cup\left(X_{2}+d\right) \text { and } Y_{1}=[0, d-1] \cup\left(Y_{2}+d\right) .
\end{aligned}
$$

We denote the equivalence class containing $\Lambda$ by $[\Lambda]$.

(b) The defect of a symbol $\Lambda=\{X, Y\}$ is defined by $\operatorname{def}(\Lambda)=|| X|-| Y||$, and the rank of $\Lambda=\{X, Y\}$ is given by

$$
r k(\Lambda)=\sum_{x \in X} x+\sum_{y \in Y} y-\left\lfloor\left(\frac{|X|+|Y|-1}{2}\right)^{2}\right\rfloor .
$$

(c) A cohook $\nu$ of a symbol $\Lambda=\{X, Y\}$ is a pair $(y, x) \in \mathbb{N}_{0}^{2}$ with $0 \leq y<x$ and

$$
\begin{array}{rll}
y \notin Y & \text { and } & x \in X \\
\text { or } y \notin X & \text { and } & x \in Y .
\end{array}
$$

Then $x-y=: k$ is called the length of $\nu$ which is then called a $k$-cohook. The symbol $\Lambda^{\prime}$ obtained from deleting $x$ from $X$ (resp. $Y$ ) and adding $y$ to $Y$ (resp. $X)$ is said to be obtained from $\Lambda$ by removing $\nu$. The $e^{\prime}$-cocore $\Lambda_{\infty}$ of $X$ is the unique symbol obtained by deleting $e^{\prime}$-cohooks as often as possible. If $\Lambda$ is degenerate and $\Lambda \neq \Lambda_{\infty}$, both copies of $\Lambda_{\infty}$ are considered as the $e^{\prime}$-cocore of $\Lambda$ (see [FS90]).

Definition 5.2 Let $\Lambda=\{X, Y\}$ be a symbol and $e^{\prime}$ be a positive integer. By an $e$-abacus we mean an abacus with $e=2 e^{\prime}$ strings numbered from left to right by $0_{l}, \ldots,\left(e^{\prime}-1\right)_{l}, 0_{r}, \ldots,\left(e^{\prime}-1\right)_{r}$ and from top to bottom by $0,1, \ldots$ With $\Lambda$ we associate an abacus diagram, called the e-unitary diagram, obtained as follows: For a positive integer $t$ we put a bead on the $i_{l}$-th string of row number $t$ if $t$ is even and if $i+t e^{\prime}=: x^{\prime}$ is an element of $X$ or if $t$ is odd and $x^{\prime}$ is an element of $Y$. Analogously we put a bead on string $i_{r}$ and row number $t$ if $t$ is even and $i+t e^{\prime}=x^{\prime}$ is an element of $Y$ or if $t$ is odd and $x^{\prime}$ is an element of $X$. In these cases we say the bead has number $x_{l}^{\prime}$ and $x_{r}^{\prime}$, respectively. The bead sticking last on its string is called index-bead. 
Note that removing an $e^{\prime}$-cohook $\nu=(y, x)$ from a symbol $\Lambda$ means pushing the bead $x$ up to the (free) position $y$ lying above $x$.

By ([Ca85, Sec. 13.8]) we have for a positive integer $\tilde{m}$ :

Remark 5.3 The unipotent characters of $S p_{2 \tilde{m}}(q)$ are parametrized by equivalence classes of symbols of rank $\tilde{m}$ and odd defect.

The unipotent characters of $S O_{2 \tilde{m}}^{+}(q)$ are parametrized by equivalence classes of symbols of rank $\tilde{m}$ and defect divisible by 4 .

The unipotent characters of $S O_{2 \tilde{m}}^{-}(q)$ are parametrized by equivalence classes of symbols of rank $\tilde{m}$ and defect congruent to 2 modulo 4 .

In what follows we need to take results from the theory of Deligne-Lusztig (see also [Ca85, Sec. 13.7, 13.8]).

Theorem 5.4 Let $\tilde{m}$ be a positive integer and $\chi_{\Lambda}$ be a unipotent character parametrized by the symbol $\Lambda$. Then $\chi_{\Lambda}$ is cuspidal if and only if

(a) for $G_{2 \tilde{m}}(q)=S p_{2 \tilde{m}}(q)$, it is equivalent to $\{\{0,1, \ldots, 2 r\},\{\}\}$ for some positive integer $r$ (and $\left.\tilde{m}=r^{2}+r\right)$,

(b) for $G_{2 \tilde{m}}(q)=S O^{\varepsilon}(q)(\varepsilon= \pm 1)$, it is equivalent to $\{\{0,1, \ldots, 2 r-1\},\{\}\}$ for some positive integer $r$ (and $\tilde{m}=r^{2}$ with $r$ odd if $\varepsilon=-1$, and $r$ even otherwise).

In these cases the cuspidal character is unique.

Lemma 5.5 Let $s \neq t$ be positive integers with $0<t<e^{\prime}$ and $s+t=2 e^{\prime}$. Then there is a symbol $\Lambda=\{X, Y\}$, such that the corresponding e-unitary diagram has a fully occupied first row, $s$ index-beads corresponding to $X$ and $t$ index-beads corresponding to $Y$.

Proof: We start with beads for $X$ and $Y$ at string $0_{l}$ and $0_{r}$, respectively.

Put a bead on each position of the first row. Then for each $e^{\prime} \leq i \leq\left(e^{\prime}-1-t\right)$ put a bead on each position $i_{r}$. Then there are no beads on row number 1 at $t$ positions within the right part of the abacus.

Given $s, t$, we let for the rest of this chapter $\Lambda=\{X, Y\}$ be as in Lemma 5.5 with $|X|>|Y|$ and label its index-beads such that $\sigma_{1}>\sigma_{2}>\cdots>\sigma_{s}$ correspond to $X$ and $\tau_{1}>\tau_{2} \cdots>\tau_{t}$ correspond to $Y$. We put $m:=r k(\Lambda)+e^{\prime}$. Considering Remark 5.3 we fix $S p_{2 m}(q)$, if $\operatorname{def}(\Lambda)$ is odd, $S O_{2 m}^{+}(q)$ if $\operatorname{def}(\Lambda)$ is divisible by 4 or $S O_{2 m}^{-}(q)$ if $\operatorname{def}(\Lambda)$ is even and not divisible by 4 . Note, that the corresponding subgroup $S p_{2\left(m-e^{\prime}\right)}(q), S O_{2\left(m-e^{\prime}\right)}^{+}(q)$ or $S O_{2\left(m-e^{\prime}\right)}^{-}(q)$ has a cuspidal unipotent character by Theorem 5.4. By condition (4) we observe that $\left|p^{f}\right|_{l}=2 e^{\prime}=$ : $e$ and $\left|-p^{f}\right|_{l}=$ $e^{\prime}$. Hence, by [FS82], $l$ is unitary for $G_{2 m}$ and the cyclic unipotent $l$-blocks of $G_{2 m}$ are parametrized by $e^{\prime}$-cocores $\Lambda$. Assume $B_{\Lambda}$ to be a cyclic unipotent block parametrized by $\Lambda$. By [FS90] we have:

Theorem 5.6 The symbols $\Lambda_{\sigma_{i}}$ and $\Lambda_{\tau_{j}}$ obtained from $\Lambda$ by adding the cohooks $\left(\sigma_{i}, \sigma_{i}+e^{\prime}\right)$ and $\left(\tau_{j}, \tau_{j}+e^{\prime}\right), 1 \leq i \leq s$ and $1 \leq j \leq t$ label the non-exceptional characters of $B_{\Lambda}$, denoted by $\chi_{\sigma_{i}}$ and $\chi_{\tau_{j}}$, respectively. Then $s=e^{\prime}+\operatorname{def}(\Lambda)$ and $t=$ $e^{\prime}-\operatorname{def}(\Lambda)$. The Brauer tree of $B_{\Lambda}$ is of the following shape with the exceptional character between $\chi_{\sigma_{s}}$ and $\chi_{\tau_{t}}$ :

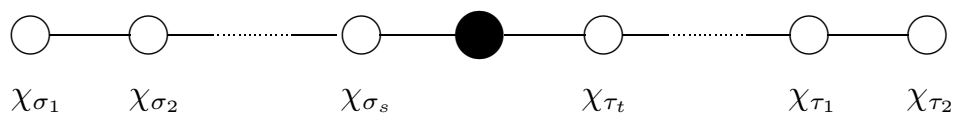

As this Brauer tree is the basic tree we first need to ensure that the symbol $\Lambda$ exists for all choices of $s$ and $t$ with $s+t=2 e^{\prime}$. 


\subsection{The Basic Tree}

We need to analyse the structure of the occurring defect groups. Let the notation be as in the last section.

Lemma 5.7 Let $\tilde{m}>\tilde{e}$ be positive integers.

(a) There is a subgroup of $S p_{2 \tilde{m}}(q)$ of the form $S p_{2 \tilde{e}}(q) \times S p_{2(\tilde{m}-\tilde{e})}(q)$. Moreover there is an irreducible cyclic subgroup of $S p_{2 \tilde{m}}(q)$ of order $q^{\tilde{m}}+1$ and each irreducible cyclic subgroup of $\operatorname{Sp}_{2 \tilde{m}}(q)$ has an order dividing $q^{\tilde{m}}+1$.

(b) There is a subgroup of $S_{2 \tilde{m}}^{-}(q)$ of the form $S O_{2 \tilde{e}}^{-}(q) \times S O_{2(\tilde{m}-\tilde{e})}^{+}(q)$. Moreover there is an irreducible cyclic subgroup of $S O_{2 \tilde{m}}^{-}(q)$ of order $q^{\tilde{m}}+1$ and each irreducible cyclic subgroup of $S_{2 \tilde{m}}^{-}(q)$ has a order dividing $q^{\tilde{m}}+1$.

(c) There is a subgroup of $S O_{2 \tilde{m}}^{+}(q)$ which is isomorphic to $S O_{2 \tilde{e}}^{-}(q) \times S O_{2(\tilde{m}-\tilde{e})}^{\varepsilon}$ for $\varepsilon \in\{+,-\}$.

Proof: See [Hup70].

Given $s, t$, let $\Lambda, m$ and $e^{\prime}$ be as in the last subsection. Then we denote the subgroups of $G_{2 m}$ introduced above by $G_{2 e^{\prime}}, G_{2\left(m-e^{\prime}\right)}$ and $G_{2 e^{\prime}} \times G_{2\left(m-e^{\prime}\right)}$. Fix a cyclic irreducible subgroup $T_{0}$ in $G_{2 e^{\prime}}$ and the Sylow $l$-subgroup $D_{0}$ of $T_{0}$ which is, by condition (4), also a Sylow $l$-subgroup of $G_{2 e^{\prime}}$. By Theorem 4.4(ii) in [CaEn94], a Sylow $l$-subgroup of $G_{2 e^{\prime}}$ is a defect group for the unipotent block $B_{\Lambda}$. In particular $B_{\Lambda}$ has $T_{0}$ as defect group.

Lemma 5.8 (a) We have $C_{G_{2 e^{\prime}}}\left(T_{0}\right)=T_{0}$ and $C_{G_{2 m}}\left(T_{0}\right)=T_{0} \times G_{2\left(m-e^{\prime}\right)}=$ $C_{G_{2 m}}\left(D_{0}\right)$,

(b) $N_{G_{2 m}}\left(T_{0}\right)=N_{G_{2 e^{\prime}}}\left(T_{0}\right) \times G_{2\left(m-e^{\prime}\right)}=T \rtimes C_{2 e^{\prime}} \times G_{2\left(m-e^{\prime}\right)}$, with a cyclic group $C_{2 e^{\prime}}$ of order $2 e^{\prime}$ and

(c) $N_{G_{2 m}}\left(D_{0}\right)=N_{G_{2 m}}\left(T_{0}\right)$.

Proof: Analogously to Lemma 4.7 we get (a), (b), (d) and the first part of (c). For the second part of (c) compare [Ca85, Prop. 3.3.6] and [Ca85, Cor. 3.6.5].

\subsection{Unfolding the Basic Tree}

We now consider extensions of the occurring groups. Let $B_{\Lambda}, T_{0}, D_{0}$ and $\chi_{\Lambda}$ be as above. Obviously the restricted Frobenius automorphism $\alpha:=\bar{\alpha}_{\mid \mathbb{F}_{q}}$ acts on $S p_{2 m}(q)$ and $S O_{2 m}^{+}(q)$. It remains to define a suitable action on $S O_{2 m}^{-}(q)$.

Remark 5.9 Let $V$ be a $2 m$-dimensional vector space over $\mathbb{F}_{q}$ with quadratic form $Q$ of Witt index $m-1$ such that $S_{2 m}^{-}(q)$ is isomorphic to the corresponding orthogonal group. Let the polynomial $X^{2}+X+a \in \mathbb{F}_{q}[X]$ be irreducible and $\left(v_{1}, \ldots, v_{m}, v_{m}^{\prime}, \ldots, v_{1}^{\prime}\right):=\left(\tilde{v}_{1}, \ldots, \tilde{v}_{2 m}\right)$ be a basis of $V$ such that

$$
Q\left(\sum_{i=1}^{2 m} x_{i} \tilde{v}_{i}\right)=\sum_{i=1}^{m-1} x_{i} x_{2 m+1-i}+x_{m}^{2}+x_{m} x_{m+1}+a x_{m+1}^{2} .
$$

Then, for $0 \leq r \leq f, X^{2}+X+\alpha^{i}(a) \in \mathbb{F}_{q}[X]$ is irreducible, too. Thus the following quadratic forms $Q_{r}$ are isometric to $Q$ :

$$
Q_{r}\left(\sum_{i=1}^{2 m} x_{i} \tilde{v}_{i}\right)=\sum_{i=1}^{m-1} x_{i} x_{2 m+1-i} x_{m}^{2}+x_{m} x_{m+1}+\alpha^{-r}(a) x_{m+1}^{2} .
$$


Hence, for each $0 \leq r \leq f$, there is an automorphism $\psi_{r} \in \operatorname{Aut}(V)$ corresponding to the isometry between $(V, Q)$ and $\left(V, Q_{r}\right)$. Then $\alpha^{\prime}:=\left(\alpha, \psi_{1}\right)$ acts on $S O_{2 m}^{-}(q)$ by mapping $\left[g_{i, j}\right]_{1 \leq i, j \leq 2 m} \in S O_{2 m}^{-}(q)$ to

$$
\alpha^{\prime}\left(\left[g_{i j}\right]_{1 \leq i, j \leq 2 m}\right)=\alpha\left(\psi\left(\left[g_{i j}\right]_{1 \leq i, j \leq 2 m}\right)\right) .
$$

Let the notation be as above. We use $\alpha^{(*)}$ to denote $\alpha$ and $\alpha^{\prime}$ depending on which group $G_{2 m}$ is considered. Put

$$
\hat{G}_{2 m}:=G_{2 m} \rtimes\left\langle\alpha^{(*)}\right\rangle .
$$

Then $\left|\hat{G}_{2 m}: G_{2 m}\right|=\left|\alpha^{(*)}\right|=f$, which is not divisible by $l$. As $\alpha^{(*)}\left(T_{0}\right) \leq G_{2 e^{\prime}}$ is again a Coxeter torus, we find $g_{0} \in G_{2 e^{\prime}}$ such that $g_{0}\left(\alpha^{(*)}\left(T_{0}\right)\right) g_{0}^{-1}=T_{0}$. Put $\sigma:=$ $\left(g_{0}, \alpha^{(*)}\right) \in \hat{G}_{2 m}$. Then $\hat{G}_{2 m}=G_{2 m}\langle\sigma\rangle$. Then $T_{0}, D_{0}, G_{2 m}, G_{2\left(m-e^{\prime}\right)}(q), C_{G_{2 m}}\left(D_{0}\right)$ and $N_{G_{2 m}}\left(D_{0}\right)$ are invariant under $\sigma$.

With the relations (4) we have $C_{\hat{G}_{2 m}}\left(D_{0}\right)=C_{G_{2 m}}\left(D_{0}\right)=T_{0} \times G_{2\left(m-e^{\prime}\right)}$. By [Alp93, Thm. 15.1(5)], there is exactly one block $\hat{B}$ of $\hat{G}_{2 m}$ which covers $B_{\Lambda}$ with $\hat{B}=$ $\left(B_{\Lambda}\right)^{\hat{G}_{2 m}}$. The same argumentation as in Lemma 4.9 shows that, $\chi_{\Lambda}$ is invariant under $\sigma_{\mid G_{2\left(m-e^{\prime}\right)}}$. It remains to analyse the inertia group of the canonical character to determine the number of edges of the Brauer tree.

Lemma 5.10 We have $T_{N_{\hat{G}_{2 m}}\left(D_{0}\right)}\left(1_{T_{0}} \otimes \chi_{\Lambda}\right)=N_{\hat{G}_{2 m}}\left(D_{0}\right)$.

Proof: As $N_{G_{2 m}}\left(D_{0}\right)$ is invariant under $\sigma$ we have $N_{\hat{G}_{2 m}}\left(D_{0}\right)=\left\langle N_{G_{2 m}}\left(D_{0}\right), \sigma\right\rangle$. Moreover $1_{T_{0}} \otimes \chi_{\Lambda}$ is invariant under $\sigma$ and under $N_{G_{2 m}}\left(D_{0}\right)$.

Hence the inertia index is $\left|T_{N_{\hat{G}_{2 m}}\left(D_{0}\right)}\left(\lambda_{0}\right): C_{\hat{G}_{2 m}}\left(D_{0}\right)\right|=f \cdot 2 e^{\prime}$ and the block under consideration has $2 e^{\prime} f=e f$ edges. By [Fei84, La. 3.2] we have $S_{s, t, f}$ as Brauer tree for $\hat{B}$. By the same arguments as in Remark 4.11, this Brauer tree is also the planar embedding.

\section{Brauer Trees in Principal Blocks}

In this section we discuss the question whether there are Brauer trees which cannot be realized in the principal block of any group. Indeed we find in Theorem 2 an infinite family of Brauer trees which do not occur in the principal block of any group. To reduce the assertion of Theorem 2 to simple groups, we need the following lemma.

Lemma 6.1 Let $G$ be a group with a cyclic Sylow l-subgroup and with $O_{l^{\prime}}(G)=\{1\}$.

(a) The product $S$ over all minimal normal subgroups of $G$ is simple with $l|| S \mid$.

(b) $G$ is solvable or satisfies $S \leq G \leq \operatorname{Aut}(S)$.

\section{Proof:}

(a) Assume that $M_{1} \neq M_{2}$ are minimal normal subgroups of $G$. Then $l|| M_{1} \mid$ and $l|| M_{2} \mid$, since $O_{l^{\prime}}(G)=\{1\}$. Consider subgroups $T_{i} \leq M_{i}$ of order $l$ for $i=1,2$. As $G$ has a cyclic Sylow $l$-subgroup, there a is $g \in G$ with $g^{-1} T_{1} g=T_{2}$. Since $M_{1}$ is normal, $M_{1} \cap M_{2} \neq\{1\}$, which is a contradiction to the minimality of $M_{i}$. Hence there is a unique minimal normal subgroup $S$, which is simple since it has a cyclic Sylow $l$-subgroup.

(b) Let $C:=C_{G}(S)$. If $S$ is abelian, it is cyclic of order $l$. Let $[C, C]$ denote the commutator of $C$. By [Isa94, Cor 5.6], $S \cap[C, C]=\{1\}$. As $S$ is the unique minimal normal subgroup, we have $[C, C]=\{1\}$, hence $C$ is abelian. As $G / C$ 
embeds into $\operatorname{Aut}(S) \cong C_{l-1}$, it follows that $G$ is solvable. If $S$ is non-abelian, then $C \cap S=\{1\}$. As $C$ is a normal subgroup of $G$, we have $C=\{1\}$ by the minimality and uniqueness of $S$ as a minimal normal subgroup of $G$. Hence, $S \leq G=N_{G}(S) \leq \operatorname{Aut}(S)$. Note that in this case, $l \nmid|G: S|$ by [Ga52].

The previous lemma provides the reduction to prove Theorem 2 .

Proof of Theorem 2: We show that most irregularly shaped stars do not occur as Brauer trees of principal blocks of any group. Let $G$ be an arbitrary group. Since we consider the principal block, we may assume that $O_{l^{\prime}}(G)=\{1\}$. Note that if $S_{s, t, f}$ and $S_{s^{\prime}, t^{\prime}, f^{\prime}}$ are similar stars with $s<t$ and $s^{\prime}<t^{\prime}$, then $s=s^{\prime}$ and $t=t^{\prime}$. If $G$ is solvable then the Brauer tree is a regularly shaped star with one edge on each ray. Thus it cannot be similar to an irregularly shaped star. By Lemma 6.1, we henceforth may assume that $S \leq G \leq \operatorname{Aut}(G)$ for some non-abelian simple group $S$. It therefore suffices to prove the Theorem for non-abelian simple groups by [Fei84, La. 4.3]. We consider these in turn.

(a) $A_{n}$ for $n \geq 5$ : If $A_{n}$ has a cyclic Sylow $l$-subgroup, the Brauer tree of the principal block is a straight line with the exceptional vertex sitting on one end (see [Ja78]).

(b) Groups of Lie type:

- $P S L_{n}(q), n \geq 2, n \neq(2,2),(2,3)$ : If $l \mid q$, we only need to consider $n=2$ and $q=l$. In this case the principal block of $P S L_{2}(l)$ is isomorphic to the principal block of $S L_{2}(l)$, which is a basic tree for a regularly shaped star by [Alp93, Chap.V, p.123]. If $l \mid q-1$, we only need to consider $n=2$. From $[\mathrm{Bu} 76]$ we know, that in this case the Brauer tree of the principal block of $P S L_{2}(q)$ is a basic tree for a regularly shaped star. It remains to consider the case $l \nmid q$ and $l \nmid q-1$. As above, the principal $l$-block of $P S L_{n}(q)$ is isomorphic to the principal block of $S L_{n}(q)$. By [Fei84] the principal block of $S L_{n}(q)$ is in this case similar to the principal block of $G L_{n}(q)$, which in turn has a basic tree for a regularly shaped star by [FS84].

- $P S U_{n}\left(q^{2}\right), n \geq 3,(n, q) \neq(3,2)$ : The same argumentation as in case $P S L_{n}(q)$ shows, that we may consider $G U_{n}\left(q^{2}\right)$, and the results in Section 4 complete the proof in this case.

- $P S p_{n}(q), n \geq 2, n \neq(2,2)$ : Let the $l$-Sylow sugroups of $P S p_{n}(q)$ be cyclic. As $\left|\bar{Z}\left(S p_{n}(q)\right)\right|=\operatorname{gcd}(2, q-1)$, we may apply the results of Section 5 , unless $l=2$. But in this case all occurring Brauer trees only have one edge.

- $\Omega_{2 m+1}(q), n \geq 3, q$ odd: We may consider $G O_{2 m+1}(q)$ and apply the results of Section 5, we may assume $l$ to be odd.

- $\Omega_{2 m}^{+}(q), \Omega_{2 m}^{-}(q), n \geq 4$. Assume $l$ to be odd. Then we may consider $G O_{2 m}^{+}(q)$ and $G O_{2 m}^{-}(q)$, respectively, and apply the results of Section 5 .

- $E_{6}(q)$ : This group has a basic tree for an irregularly shaped star with parameters $(s, t, f)=(2,6, f)$ with $8 f \mid l-1$ and $l \mid q^{4}+1$ in its principal block by [HiLueMal95].

- $E_{7}(q), E_{8}(q)$ : The Brauer trees of those groups have less than 249 edges (see [Fei84]).

- $F_{4}(q)$ : This group provides no basic tree for an irregularly shaped star in the principal block by [HiLue98].

- ${ }^{3} D_{4}\left(q^{3}\right)$ : There is one basic tree for a star with parameters $(s, t, f)=$ $(1,3, f)$ with $f \mid l-1$ and $4 l \mid q^{4}-q^{2}+1$ in its principal block by [Ge90]. 
- ${ }^{2} E_{6}\left(q^{2}\right)$ : This group has a basic tree for an irregularly shaped star with $(s, t, f)=(1,4, f)$ with $5 f \mid l-1$ and $l \mid q^{4}+q^{3}+q^{2}+q+1$ in its principal block by [HiLue98].

- $G_{2}(q),{ }^{2} B_{2}\left(q^{2}\right),{ }^{2} F_{4}\left(q^{2}\right),{ }^{2} G_{2}\left(q^{2}\right)$ : There are no exceptions by [Hi90].

(c) Sporadic groups: By [HiLux89] there are only two principal $l$-blocks with Brauer trees $S_{s, t, f}$ : In $J_{1}$ for $l=19$ the Brauer tree equals $S_{1,5,1}$ and in $J_{4}$ for $l=31$, the Brauer tree equals $S_{3,7,1}$

\section{Acknowledgments}

I am very grateful to Gerhard Hiss for his valuable suggestions and steady help. The results discussed here are part of my Diploma Thesis.

\section{References}

[Alp93] J. L. Alperin, Local representation theory, Cambridge University Press, 1. Paperback edition, New York, 1993.

[Bu76] R. Burkhardt, Zerlegungsmatrizen der PSL(2, $\left.p^{f}\right)$, J. Algebra 40 (1976), 75-96.

[CaEn94] M. Cabanes and M. Enguehard, On unipotent blocks and their ordinary characters, Invent. Math. 117 (1994), 149-164.

[Ca85] R. W. Carter, Finite groups of Lie type, John Wiley \& Sons Ltd. 1., Edition Chichester, 1985.

[FS82] P. Fong and B. Srinivasan, The blocks of finite general linear and unitary groups, Invent. Math. 69 (1982), 109-153.

[FS84] P. Fong and B. Srinivasan, Brauer trees in $G L(n, q)$, Math. Z. 187 (1984), 81-88.

[FS90] P. Fong and B. Srinivasan, Brauer trees in classical groups, J. Algebra 131(1990), 179-225.

[Fei82] W. Feit, Representation theory of finite groups, North-Holland Publishing Company, Amsterdam, 1982.

[Fei84] W. Feit, Possible Brauer trees, Illinois J. Math. 28 (1984), 43-54.

[Ga52] W. Gaschütz, Zur Erweiterungstheorie endlicher Gruppen, J. f. d. reine und angew. Math. 190(1952), 93-107.

[Ge90] M. Geck, Verallgemeinerte Gelfand-Graev Charaktere und Zerlegungszahlen endlicher Gruppen vom Lie-Typ, Dissertation, Aachen, 1990.

[Has64] H. Hasse, Vorlesungen über Zahlentheorie, Springer-Verlag, 2nd. Edition, Berlin, 1964.

[Hi90] G. Hiss, Zerlegungszahlen endlicher Gruppen vom Lie Typ in nicht-definierender Charakteristik, Habilitationsschrift, Aachen, 1990.

[HiLue98] G. Hiss, F. Lübeck, The Brauer trees of the exceptional Chevalley groups of type $F_{4}$ and ${ }^{2} E_{6}$, Arch. d. Math.70(1998), 16-21. 
[HiLueMa195] G. Hiss, F. Lübeck, G. Malle, The Brauer trees of the exceptional Chevalley group of type $E_{6}$, Manuscripta Math. 87(1995), 131-144.

[HiLux89] G. Hiss, K. Lux, Brauer Trees of Sporadic Groups, Oxford University Press, New York, 1989.

[Hup67] B. Huppert, Endliche Gruppen I, Springer-Verlag, Heidelberg, 1967.

[Hup70] B. Huppert, Singer-Zyklen in klassischen Gruppen, Math. Z. 117 (1970), 141-150.

[HupBla82] B. Huppert and N. Blackburn, Finite Groups II, Springer-Verlag, Heidelberg, 1982.

[Isa94] I.M. Isaacs, Character Theory of Finite Groups, Dover Publications, Mineola, 1994.

[Ja78] G.D. James, The representation theory of the symmetric group, Lecture Notes in Math., Vol. 682, Springer Verlag, 1978.

[KSt98] H. Kurzweil and B. Stellmacher, Theorie der endlichen Gruppen, SpringerVerlag, Heidelberg, 1998.

[Lu77] G. Lusztig, Representations of finite classical groups, Invent. Math. 43 (1977), 125-175.

[Lu84] G. Lusztig, Characters of reductive groups over a finite field, Ann. Math. Stud. 107(1984), Princeton Univ. Press, Princeton, NJ. 